\title{
Diagnostic Value of Diffusion-Weighted Whole-Body Imaging with Background Body Signal Suppression for the Detection of Acute Pyelonephritis in Children: Case-Control Study Yasutaka Kuniyoshi*, Azusa Kamura, Sumie Yasuda and Makoto Tashiro
}

Department of Pediatrics, Tsugaruhoken Medical CO-OP Kensei Hospital, Japan

\begin{abstract}
Background: The objective of this study was to investigate the accuracy of diffusion-weighted whole-body imaging with background body signal suppression (DWIBS) as a diagnostic test for acute pyelonephritis (APN) in children.

Methods: Among inpatients under 15 years of age in a primary care setting, we retrospectively reviewed the medical records for 29 febrile children subjected to DWIBS to confirm or exclude APN. To assess the diagnostic value of DWIBS, the subjects were divided into 13 patients clinically diagnosed with APN and 16 non-APN patients. DWIBS findings were divided into positive, indeterminate, and negative based on visual observation.
\end{abstract}

Results: Likelihood ratios for positive, indeterminate, and negative prediction were 6.15 (95\% Cl, 1.90-19.97), 0.49 $(95 \% \mathrm{Cl}, 0.13-1.83)$ and $0.14(95 \% \mathrm{Cl}, 0.03-0.65)$, respectively.

Conclusions: This study is the first to estimate the accuracy of DWIBS for the detection of APN. Initial DWIBS is a sensitive method for the diagnosis of APN and can improve diagnostic performance.

Keywords: Acute pyelonephritis; Diffusion-weighted whole-body imaging with background body signal suppression; Stratum-specific likelihood ratios

\section{Background}

Urinary tract infection (UTI) is a common bacterial infection in infants and children. If UTI is not detected and left untreated, it may lead to permanent kidney damage; kidney scarring, hypertension, and chronic kidney impairment. Therefore, appropriate diagnosis and treatment of UTIs is important. Diagnosis of acute pyelonephritis (APN) is usually based on clinical and biological data, because there is no gold standard. The clinical and biological findings of patients with UTI are of limited value [1]. The diagnosis of APN is not based on urine culture alone. The role of diagnostic imaging in the evaluation of patients suspected of UTI has been debated [2]. It is reported that kidney scanning with technetium-99 m-labeled dimercaptosuccinic acid (DMSA) scintigraphy is useful as a diagnostic test for APN [3]. However, this technology is not readily accessible in all hospitals and there is no consensus on its routine use [4].

Diffusion-weighted whole-body imaging with background body signal suppression (DWIBS) recently introduced by Takahara [5] has been shown to be useful not only for the detection not only of oncological lesion but also for that of non-oncological lesions [6].

The objective of this study was to evaluate the accuracy of DWIBS as a diagnostic test for APN.

\section{Methods \\ Patient}

We retrospectively reviewed the medical records for febrile $(\geq$ $38.5^{\circ} \mathrm{C}$ ) children under 15 years old of age who were hospitalized in our department from January 2011 to December 2012, and were subjected to DWIBS to confirm or exclude acute pyelonephritis. Patients with congenital abnormalities of the kidneys were excluded. To assess the diagnostic value of DWIBS, the subjects were divided into 13 patients clinically diagnosed with APN and 16 non-APN patients. Patients with two or more of the following findings and without other disorders were defined as APN:

1) White blood count $(\mathrm{WBC}) \geq 15,000$ and/or C-reactive protein $(\mathrm{CRP}) \geq 3.0 \mathrm{mg} / \mathrm{dL}$;

2) Pyuria ( $\geq 10$ white blood cells/high-power field on the urinary analysis), and

3) Positive urine culture result ( $\geq 105$ colony-forming-units $/ \mathrm{mL}$ of a single urinary tract pathogen).

The method of urine collection was clean catch or bag specimen: $<36$ month patients were enrolled for urine bag sampling and $\geq 36$ month patients were for clean catch sampling. In the assessment of the urine culture results, common contaminants, including Lactobacillus spp., Corynebacterium spp., coagulase-negative staphylococci, and a-hemolytic streptococci, were excluded.

\section{DWIBS findings}

Magnetic resonance imaging (MRI) was performed with a 1.5-Tesla system (Philips Healthcare, The Netherlands), using a surface coil (SENSE body coil $1.5 \mathrm{~T} 4 \mathrm{ch}$, Philips Healthcare). DWIBS for all cases was performed within 5 days of admission.

DWIBS findings were visually determined and classified as positive, indeterminate, or negative by at least two pediatricians. For example, Figure 1 represents a positive DWIBS result.

*Corresponding author: Yasutaka Kuniyoshi, Department of Pediatrics Tsugaruhoken Medical CO-OP Kensei Hospital, 2-2-1 Noda, Hirosaki, Aomori, Japan, 036-8511, Tel: +81172321171; Fax: +81-172-35-1678; E-mail: yasutakakuniyoshi@yahoo.co.jp

Received: July 22, 2015; Accepted: August 23, 2015; Published: August 30, 2015

Citation: Kuniyoshi Y, Kamura A, Yasuda S, Tashiro M (2015) Diagnostic Value of Diffusion-Weighted Whole-Body Imaging with Background Body Signal Suppression for the Detection of Acute Pyelonephritis in Children: Case-Control Study. J Nephrol Ther 5: 210. doi:10.4172/2161-0959.1000210

Copyright: (c) 2015 Kuniyoshi Y. This is an open-access article distributed unde the terms of the Creative Commons Attribution License, which permits unrestricted use, distribution, and reproduction in any medium, provided the original author and source are credited. 
Citation: Kuniyoshi Y, Kamura A, Yasuda S, Tashiro M (2015) Diagnostic Value of Diffusion-Weighted Whole-Body Imaging with Background Body Signal Suppression for the Detection of Acute Pyelonephritis in Children: Case-Control Study. J Nephrol Ther 5: 210. doi:10.4172/2161-0959.1000210

Page 2 of 4

\section{Statistical analysis}

Statistical analyses were performed using R version 3.0.3. Fisher's exact test for categorical variables and $t$ test for continuous variables were used to determine statistical differences. The stratum-specific likelihood ratios (SSLRs) with 95\% confidence intervals (CI) were calculated.

\section{Results}

\section{Characteristics and diagnoses of patients}

Table 1 show the clinical and laboratory findings of APN and non-APN patients. There were no statistically significant differences in age, gender, WBC count, or serum CRP value between the groups. Final diagnoses of non-APN patients were exanthema subitum (three cases), juvenile idiopathic arthritis (two cases), Streptococcus agalactiae septicemia (one case), cervical lymphadenitis (one case), adverse event related to vaccination (one case), viral infection (three cases; adenovirus, rotavirus, another virus), and fever without localizing

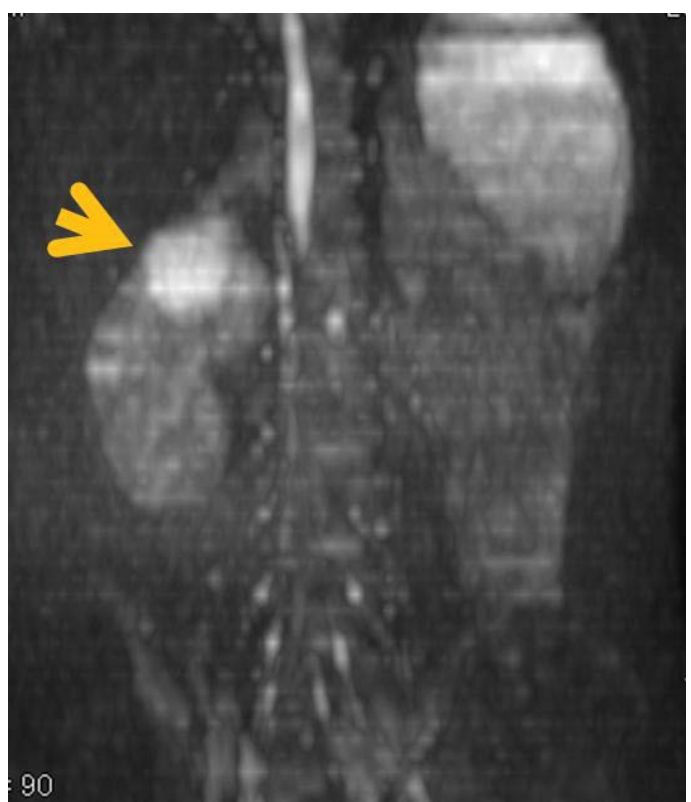

Figure 1: A 9-year-old female (subject 13 in Table 2). DWIBS (b value, $1000 \mathrm{~s} /$ $\mathrm{mm}^{2}$ ) shows signal hyperintensity in the upper zone of the right kidney (arrow)

\begin{tabular}{|c|c|c|c|}
\hline & $\begin{array}{c}\text { APN (n=13) } \\
\text { mean } \pm \text { SD or } \mathbf{n}(\%)\end{array}$ & $\begin{array}{c}\text { non APN (n=16) } \\
\text { mean } \pm \text { SD or } \mathbf{n}(\%)\end{array}$ & $\boldsymbol{p}$ \\
\hline Age (month) & $19 \pm 29$ & $31 \pm 46$ & N.S \\
\hline Gender (male:female) & $4: 9$ & $9: 7$ & N.S \\
\hline WBC $\left(/ \mathrm{mm}^{3}\right)$ & $18877 \pm 5281$ & $15375 \pm 8864$ & N.S \\
\hline CRP $(\mathrm{mg} / \mathrm{dl})$ & $6.2 \pm 3.5$ & $4.7 \pm 4.0$ & N.S \\
\hline U-WBC $(>10 \mathrm{HPF})$ & $11(85)$ & $5(31)$ & $<0.05$ \\
\hline Culture $\left(>1 \times 10^{5}\right)$ & $6(46)$ & $0(0)$ & $<0.05$ \\
\hline
\end{tabular}

Table 1: Clinical and laboratory findings of APN and non-APN patients. U-WBC: Urine WBC, Culture: Urine culture. source or fever of unknown origin (four cases).

The details of the 13 APN patients are shown in Table 2. Of these, 10 had $W B C \geq 15,000,11$ had CRP $\geq 3.0$, and 12 had $\mathrm{U}-\mathrm{WBC} \geq 10$. Two had received an antibacterial agent before the diagnosis of APN. Four showed complications of vesicoureteral reflux. Of five APN patients in whom it took over $72 \mathrm{~h}$ to reduce fever, four had findings of hyperintensity by DWIBS.

\section{Diagnostic performance of DWIBS findings (Table 3)}

Of 13 APN patients, 10 (76.9\%) had positive DWIBS findings. Of 16 non-APN patients, 9 (56.3\%) had negative DWIBS findings, and $(87.5 \%)$ had indeterminate or negative DWIBS findings. SSLRs calculated for positive, indeterminate, and negative DWIBS findings were as follows: positive DWIBS findings, 6.15 (95\% CI, 1.90-19.97); indeterminate DWIBS findings, 0.49 (95\% CI, 0.13-1.83); and negative DWIBS findings, 0.14 (95\% CI, 0.03-0.65).

\section{Discussion}

APN patients have usually been clinically diagnosed on the basis of clinical and biological findings. The accuracy of DWIBS for APN detection is unknown. We analysed data from inpatients suspected of APN in a primary care setting, and evaluated the SSLRs of DWIBS as a diagnostic test for APN. We defined DWIBS findings as a three level variable because we believe that it reflects the spectrum of clinical entities progressing from APN to kidney abscess. We believe that the more progressive it is, the clearer DWIBS findings become.

The accuracy of DWIBS as a diagnostic test for APN showed performance similar to that of DMSA scintigraphy. In this study, sensitivity and specificity of DWIBS could not be calculated because DWIBS findings were classified as positive, indeterminate, and negative. However, $76.9 \%$ of APN patients had positive DWIBS findings. In the non-APN children, $56.3 \%$ had negative DWIBS findings and $87.5 \%$ had indeterminate or negative DWIBS findings. The sensitivity and specificity of DMSA scintigraphy for the detection of APN are reported to be $69.7 \%-89 \%$ and $100 \%$, respectively $[4,7,8]$. DWIBS may be considered as an effective alternative.

The reason for the highlights in the kidneys of APN patients by DWIBS is still unclear. Diffusion-weighted imaging (DWI) reveals the micromolecular motion of water. The contrast of DWI depends on the mobility or viscosity of the water molecules in tissues. Various lesions such as malignancies, lymphomas, and abscesses have high cellular arrangements with tightly packed cells. They show restricted diffusion and are highlighted by DWIBS. Pus is a thick, high-viscosity fluid comprising water, inflammation causing bacterial cells, necrotic tissue, and proteinaceous exudates $[9,10]$. The collecting system of a pyelonephrotic kidney is filled with purulent material, which is a thick, yellowish brown, adhesive fluid that comprises plasma with very high viscosity and cellularity [11]. Kidney infection can be considered as a spectrum of clinical entities progressing from APN to kidney abscess. We accordingly speculated that pyelonephrotic kidneys show high infectious-exudate signals.

The clinical advantages of DWIBS are as follows. First, DWIBS is useful in the evaluation of other infectious lesions with other sequences (T1- and T2-weighted images) at the same time. DWIBS images alone cannot distinguish APN from abscesses, hematomas, and neoplasms. The combination of DWIBS and other sequences can not only provide anatomical information but also provide functional information within a single examination. Within the range of DWIBS 
Citation: Kuniyoshi Y, Kamura A, Yasuda S, Tashiro M (2015) Diagnostic Value of Diffusion-Weighted Whole-Body Imaging with Background Body Signal Suppression for the Detection of Acute Pyelonephritis in Children: Case-Control Study. J Nephrol Ther 5: 210. doi:10.4172/2161-0959.1000210

Page 3 of 4

\begin{tabular}{|c|c|c|c|c|c|c|c|c|c|}
\hline Subject & Age $(\mathbf{m})$ & Gender & WBC $(\mathbf{m m})$ & CRP $(\mathbf{m g} / \mathbf{d L})$ & U-WBC (HPF) & Culture & DWIBS & VUR & Duration (hour) \\
\hline 1 & 2 & M & 17200 & 6.0 & $51-100$ & + & + & - & $<24$ \\
\hline 2 & 4 & M & 20300 & 1.7 & $101-500$ & - & + & - & $<24$ \\
\hline 3 & 4 & F & 17700 & 3.6 & $101-500$ & + & + - & - & $>72$ \\
\hline 4 & 6 & F & 13600 & 8.0 & $51-100$ & + & + - & + & $24-48$ \\
\hline 5 & 6 & F & 27600 & 9.2 & $21-30$ & + & + & - & $24-48$ \\
\hline 6 & 9 & M & 10700 & 1.1 & $51-100$ & + & - & - & $48-72$ \\
\hline 7 & 10 & F & 26400 & 12.2 & $>500$ & - & + & - & $48-72$ \\
\hline 8 & 12 & F & 18900 & 3.5 & $11-20$ & $-*$ & + & - & + \\
\hline 9 & 13 & M & 14000 & 4.4 & $6-10$ & + & + & + & +72 \\
\hline 10 & 14 & F & 15600 & 4.1 & $51-100$ & - & + & + & $24-48$ \\
\hline 11 & 23 & F & 26400 & 10 & Nov-20 & $-*$ & + & - & $>72$ \\
\hline 12 & 33 & F & 20700 & 10.1 & $51-100$ & + & + & - & $24-48$ \\
\hline 13 & 110 & F & 16300 & 7.2 & $101-500$ & + & + & + & $>$ \\
\hline
\end{tabular}

Table 2: Clinical and laboratory characteristics of APN patients.

U-WBC: Urine WBC, Culture: Urine Culture, VUR: Vesicoureteral Reflux, Duration: Duration of fever, ${ }^{*}$ Preceding Antimicrobial Agent

\begin{tabular}{|c|c|c|c|}
\hline & APN (n=13) & non-APN (n=16) & $\begin{array}{c}\text { Likelihood Ratios } \mathbf{9 5 \%} \\
\mathbf{C l})\end{array}$ \\
\hline Positive & 10 & 2 & $\begin{array}{c}6.15 \\
(1.90-19.97)\end{array}$ \\
\hline Indeterminate & 2 & 5 & $\begin{array}{c}0.49 \\
(0.13-1.83)\end{array}$ \\
\hline Negative & 1 & 9 & $\begin{array}{c}0.14 \\
(0.03-0.65)\end{array}$ \\
\hline
\end{tabular}

Table 3: Diagnostic performance of DWIBS findings.

scan, a focus of infections other than that of the kidney may be occasionally identified. Second, DWIBS is non-radiological. Enhanced computerized tomography (CT) may also be useful for the detection of APN [12]; however, it has the disadvantage of radiation exposure. Third, the administration of drugs or contrast agents is not required, as with contrast-enhanced CT and radioisotope. DWIBS is not only applicable to patients with allergies to contrast agents but also to those with existing kidney insufficiency. Fourth, MRI is more popular than radioisotope equipment[4]. The disadvantage of DWIBS is the necessity of sedation for infants and young children.

There were several limitations in our study. First, the number of cases was small. However, we carefully diagnosed strongly suspected APN and non-APN based on clinical and biological data. In this study, 25 patients were enrolled for urine bag sampling because catheterization of the urethra or suprapubic aspiration are relatively invasive procedures. Positive cultures obtained using bag samples are more likely to represent contamination than a true UTI. We excluded differential diagnosis with absolute certainty. Because the sensitivity of bag samples has been reported to be $88 \%$ as opposed to that of catheter samples [13], it may be useful as an exclusive diagnosis test. Second, an apparent diffusion coefficient (ADC) map which is a voxel-by-voxel representation of ADC values were not measured in this study. This is because lesions that have very long T2- values may appear bright even though they do not restrict diffusion. Therefore, ADC map should be inspected to distinguish whether DWI images is because of restricted diffusion, T2 shine-through refers to high signal produced on DWI images that is not because of restricted diffusion rather it is because of high T2 signal that "shines through" in the DWI image. T2 shine through occurs because of long decay time of T2 in some normal tissues. In APN, the kidney cortical and medullary ADC values are markedly lower than those in the normal kidney [6]. Future studies should measure the ADC map to objectively measure diffusion characteristics.

\section{Conclusion}

This study is the first to estimate the accuracy of DWIBS for the detection of APN. Initial DWIBS is a sensitive method for the early diagnosis of APN and can improve the diagnostic performance.

\section{Acknowledgment}

The authors would like to thank Enago (www.enago.jp) for the English language review.

\section{Declaration of Conflicting Interests}

The authors declared no potential conflicts of interest with respect to the research, authorship, and/or publication of this article. The authors received no financial support for the research, authorship, and/or publication of this article.

\section{References}

1. Shaikh N, Morone NE, Lopez J, Chianese J, Sangvai S, et al. (2007) Does this child have a urinary tract infection? JAMA 298: 2895-2904.

2. National Institute for Health and Care Excellence (NICE) (2007) Urinary Tract Infection in Children: Diagnosis, Treatment and Long-term Management. NICE guidelines.

3. Riccabona M, Fotter R (2006) Radiographic studies in children with kidney disorders: what to do and when. In: Hogg RJ. Taylor and Francis (eds), Disease in Children and Adolescents: A Practical Handbook. United Kingdom.

4. Jaksic E, Bogdanovic R, Artiko V, Saranovic DS, Petrasinovic Z, et al. (2011) Diagnostic role of initial renal cortical scintigraphy in children with the first episode of acute pyelonephritis. Ann. Nucl. Med 25: 37-43.

5. Takahara T, Imai Y, Yamashita T, Yasuda S, Nasu S, et al. (2004) Diffusion weighted whole body imaging with background body signal suppression (DWIBS): technical improvement using free breathing, STIR and high resolution 3D display. Radiat Med 22: 275-282.

6. Baliyan V, Das CJ, Sharma S, Gupta AK (2014) Diffusion-weighted imaging in 
Citation: Kuniyoshi Y, Kamura A, Yasuda S, Tashiro M (2015) Diagnostic Value of Diffusion-Weighted Whole-Body Imaging with Background Body Signal Suppression for the Detection of Acute Pyelonephritis in Children: Case-Control Study. J Nephrol Ther 5: 210. doi:10.4172/2161-0959.1000210

urinary tract lesions. Clin Radiol 69: 773-782.

7. Rushton HG (1997) The evaluation of acute pyelonephritis and renal scarring with technetium 99 m-dimercaptosuccinic acid renal scintigraphy: Evolving concepts and future directions. Pediatr Nephrol 11: 108-120.

8. Tseng MH, Lin WJ, Lo WT, Wang SR, Chu ML, et al. (2007) Does a normal DMSA obviate the performance of voiding cystourethrography in evaluation of young children after their first urinary tract infection? J Pediatr 150: 96-99.

9. Verswijvel G, Vandecaveye V, Gelin G, Vandevenne J, Grieten M, et al. (2002) Diffusion-weighted MR imaging in the evaluation of renal infection: preliminary results. JBR-BTR 85: 100-103.
10. Ebisu T, Tanaka C, Umeda M, Kitamura M, Naruse S, et al. (1996) Discrimination of brain abscess from necrotic or cystic tumors by diffusion-weighted echo planar imaging. Magn Reson Imaging 14: 1113-1116.

11. Chan JH, Tsui EY, Luk SH, Fung SL, Cheung YK, et al. (2001) MR diffusionweighted imaging of kidney: differentiation between hydronephrosis and pyonephrosis. Clin Imaging 25: 110-113.

12. Yoo JM, Koh JS, Han CH, Lee SL, Ha US, et al. (2010) Diagnosing acute pyelonephritis with CT, TC-DMSA SPECT, and Doppler Ultrasound: A comparative study. Korean J Urol 51: 260-265.

13. Shaw KN, Hexter D, McGowan KL, Schwartz JS (1991) Clinical evaluation of a rapid screening test for urinary tract infections in children. J Pediatr 118: 733736 